\title{
Ensuring Radiation Safety to Staff in Lymphatic Tracing and Sentinel Lymph Node Biopsy Surgery - Some Recommendations
}

\author{
Coventry BJ ${ }^{1,2 *}$, Collins $\mathrm{PJ}^{3}$, Kollias $\mathrm{J}^{1}$, Bochner $\mathbf{M}^{1}$, Rodgers $\mathrm{N}^{4}$, Gill PG ${ }^{1}$, Chatterton BE ${ }^{3}$ and Farshid $\mathrm{G}^{4}$
}

${ }^{1}$ Breast-Endocrine Surgical Oncology Unit, Department of Surgery, University of Adelaide, Royal Adelaide Hospital, Adelaide, South Australia

${ }^{2}$ Adelaide Melanoma Unit, Department of Surgery, University of Adelaide, Royal Adelaide Hospital, Adelaide, South Australia

${ }^{3}$ Nuclear Medicine, University of Adelaide, Royal Adelaide Hospital, Adelaide, South Australia

${ }^{4}$ Pathology, SA Pathology, Institute of Medical and Veterinary Science, Adelaide, South Australia

\begin{abstract}
Introduction: Lymphatic mapping and sentinel node biopsy (LM/ SNB) techniques for melanoma and breast cancer management potentially expose staff, including operating theatre personnel, radiologists, pathologists and others, to ionising radiation.

Aims: To ascertain exposure levels in a practical setting and to establish safe work practices for staff involved in the LM/ SNB procedure pathway.

Methods: Cumulative intra-procedural extremity (hands) and whole body radiation doses were recorded separately for surgeons, pathologists and couriers during standard sentinel lymph node biopsy procedures from 13 melanoma and included also radiologists in 11 breast cancer cases.

Results: The measured extremity dose for melanoma procedures was zero for surgeons and pathologists. The extremity dose for breast cancer procedures was approximately $250 \mu \mathrm{Sv}$ for surgeons, and about $10 \mu \mathrm{Sv}$ for pathologists per breast procedure if done on the day of surgery, but is otherwise negligible; zero for the radiologist; and zero for the courier. No whole body dose was detectable for any staff member.

Conclusions: Using the international limit for skin dose some 200 breast cancer procedures could be performed per annum per surgeon (at the general public radiation limit) - and 2000 breast surgical procedures (at the radiation worker limit) based on extremity doses. Radiologists, pathologists and couriers received minimal or zero radiation doses from handling breast specimens. Melanoma procedures showed no measurable dose. Some recommendations for effective safe work practices are given.
\end{abstract}

Keywords: Radiation safety; Sentinel node biopsy; Lymphatic mapping; Melanoma; Breast cancer

\section{Introduction}

With the introduction of lymphatic mapping and sentinel node biopsy (LM/ SNB) techniques using radioactive tracers for the management of melanoma and breast cancer, operating theatre personnel, pathologists and other staff are now being exposed to ionising radiation from radiopharmaceuticals.

Lymphoscintigraphy, lymphatic tracing, sentinel node biopsy, specimen transport, specimen radiology and the subsequent pathological analysis of the tissues may result in significant external and internal radiation exposure to personnel handling the specimen or in close proximity. Several studies (refs) have been presented in abstract form and also in the published literature relating to the safety of these techniques, but recommendations for practical safe handling procedures for these agents in the clinical setting following surgery may be useful for those concerned.

To put these results into perspective, LM/ SNB techniques for breast cancer using a ${ }^{99 \mathrm{~m}} \mathrm{Tc}$ colloid yields a patient effective dose of approximately $0.3 \mu \mathrm{Sv}$. This compares with doses from ${ }^{99 \mathrm{~m}} \mathrm{Tc}$ Bone Scan $(3.6 \mu \mathrm{Sv}),{ }^{123 \mathrm{~m}}$ I thyroid scan $(4.4 \mu \mathrm{Sv})$, mammogram $(0.4 \mu \mathrm{Sv})$, chest $\mathrm{x}$-ray $(0.04 \mu \mathrm{Sv})$, CT chest $(8.3 \mu \mathrm{Sv})$ or abdominal CT $(7.2 \mu \mathrm{Sv})$, return airflight London to Sydney $(0.20 \mu \mathrm{Sv})$, UK annual consumed food and drink $(0.37 \mu \mathrm{Sv})$, or one years' residence on Denver Colorado from background radiation $(0.88 \mu \mathrm{Sv})[1]$.

The study primarily aimed to measure radiation exposure to the extremity (hands) and (whole) body of the operating surgeon during melanoma [2,3] and breast [4] sentinel node biopsy procedures. Secondary aims were to assess radiation exposure further through the entire LM/ SNB procedure pathway for radiology, pathology and courier staff. Guidelines could then be devised for safe handling and processing of radioactive tissues as part of the sentinel node biopsy procedure in order to minimise radiation exposure risk for surgical and other personnel.

\section{Methods}

\section{Patients}

Informed patient consent was obtained for all procedures. No patients were pregnant.

\section{Lymphatic mapping and sentinel node biopsy}

${ }^{99 \mathrm{~m} T c}$ Antimony sulfide colloid (Lymphflo ${ }^{\mathrm{TM}}$, Adelaide, Australia)

*Corresponding author: Prof BJ Coventry, Breast-Endocrine Surgical Oncology Unit and Adelaide Melanoma Unit, Department of Surgery, University of Adelaide, Royal Adelaide Hospital, North Terrace, Adelaide, South Australia, E-mail: brendon.coventry@adelaide.edu.au

Received June 08, 2012; Accepted June 18, 2012; Published June 22, 2012

Citation: Coventry BJ, Collins PJ, Kollias J, Bochner M, Rodgers N, et al. (2012) Ensuring Radiation Safety to Staff in Lymphatic Tracing and Sentinel Lymph Node Biopsy Surgery - Some Recommendations. J Nucl Med Radiat Ther S2:008. doi:10.4172/2155-9619.S2-008

Copyright: (c) 2012 Coventry BJ, et al. This is an open-access article distributed under the terms of the Creative Commons Attribution License, which permits unrestricted use, distribution, and reproduction in any medium, provided the original author and source are credited. 
was used for all studies and $40 \mathrm{MBq}$ was injected in 4 divided doses either intradermally around the primary melanoma excision site, or peri-tumourally around the primary breast cancer. The pre-operative lymphoscintigram was performed for each case prior to Patent Blue (Guerbet, Faulding, Adelaide) dye injection, according to the same protocol as that used by our group as part of the Multicenter Selective Lymphadenectomy Trial (MSLT-I) studies [2,3] and for breast cancer [4]. All patients were injected with the radiopharmaceutical on the morning of surgery and proceeded to the operating theatre later that day, within 3-5 hours of receiving the radiopharmaceutical. Personal dosimeters were worn during the operative period from the time of commencement to completion of the procedure. Measurements at the non-dominant index finger were performed using a finger badge dosimeter (Figure 1 - right), and whole body doses were measured using a waist badge (Figure 1 - left). Sterility was preserved by careful washing of the finger-badge in antiseptic solution, and then placing it between two layers of sterile gloves using a double gloving technique (Figure 2). The pathway of the sentinel node procedure was identified from initial entry into the operating theatre, transport of the specimen from theatre to specimen radiology (breast) and to the pathology department (melanoma and breast) and steps at each stage were measured for radiation exposure levels (Figure 3).

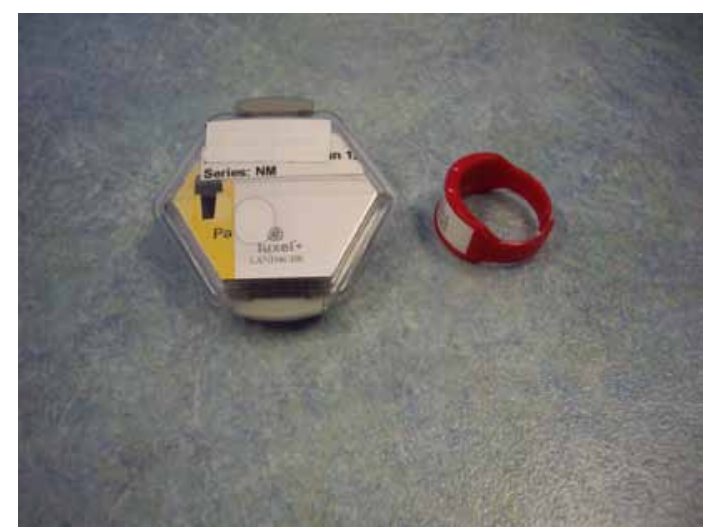

Figure 1: A Finger badge dosimeter (right), and a whole body waist badge dosimeter (left) were used for measurements of radiation exposure and used serially amongst surgeons performing procedures. Separate badges were used for radiology staff and for pathology staff performing wider excision and/ or sentinel node diagnostic procedures.

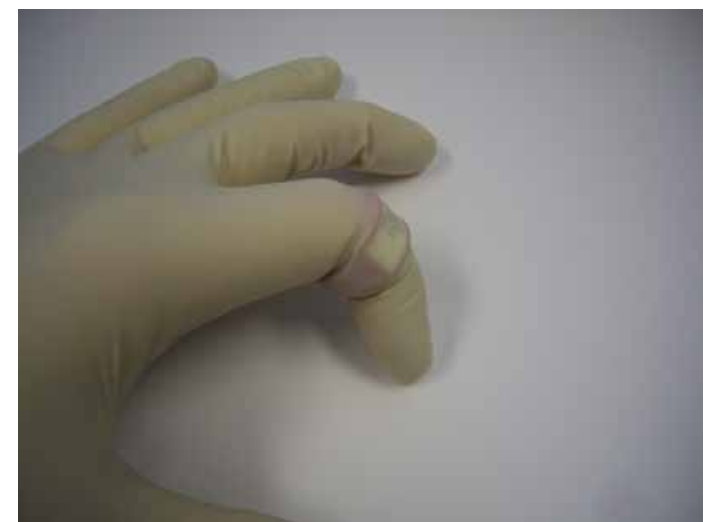

Figure 2: The finger-badge was carefully washed in antiseptic solution, and then placed between two layers of sterile gloves and worn on the non-dominan index finger, using a double gloving technique.

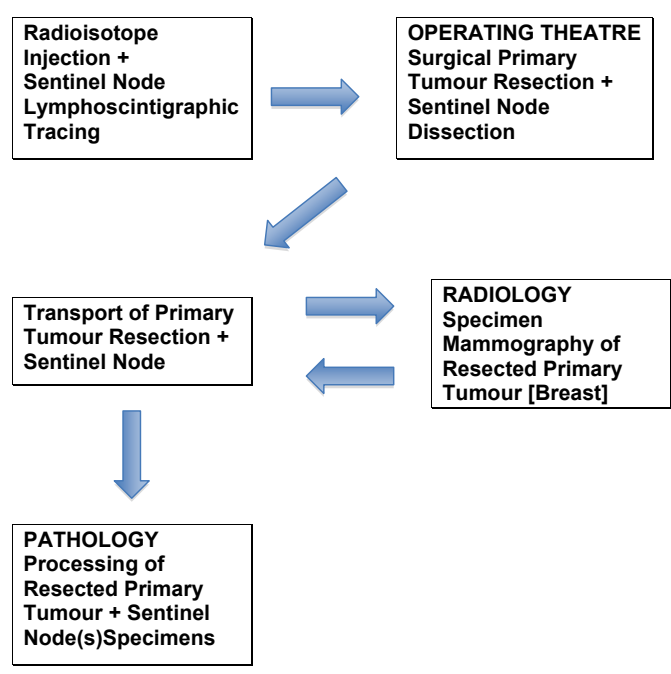

Figure 3: The pathway for the sentinel node procedure from the operating theatre, specimen transport from theatre to specimen radiology (breast) and to the pathology department (melanoma and breast) during which radiation exposure was measured.

\section{Staff radiation measurement}

Surgeons: Intraoperative radiation doses were recorded for surgeons during sentinel lymph node biopsy procedures. Thirty-six (36) procedures were used to estimate whole body radiation doses and 24 procedures for extremity (finger) doses. Four surgeons participated in the studies and wore the whole body (WB) and finger badge (FB) sequentially for estimation of cumulative doses. No surgeons were pregnant.

The radiation exposure to the whole body and extremity (hands) of the surgeon were evaluated using thermo-luminescent dosimeters (TLDs) worn on the waist/ chest and as a finger badge on the nondominant index finger respectively (Figure 1 and 2). The finger and whole body dosimeters were worn by different surgeons for sequential sentinel node operations for 1 and 3 months respectively, to provide cumulative doses. The TLD dosimetry was measured by ARPANSA (Melbourne Australia).

Specimen radiology: Whole body and extremity measurements using TLD badges were recorded in the radiology suite for the radiologist performing breast specimen radiographs and ultrasounds of excised breast tissue containing the primary tumour from $\mathrm{SN}$ procedures. This tissue included most of the injected radiopharmaceutical dose. The cumulative radiation dose to the finger and whole body of the radiologist performing the studies was measured for specimens from 28 breast cancer patients.

Pathological processing: The primary tumour specimen contained approximately $95 \%$ of the radioactivity, whereas the sentinel node only contained $<5 \%$ of the injected dose. The radiation exposure to the pathologist and technician (combined) was measured over two separate time periods using two different processing methods. In Method I the tissues ( 9 melanoma and breast cancer specimens) were processed the day following LM/ SNB surgery. Method II included imprint cytology which was performed intra-operatively at the time of the surgery on the margins of the resected breast tissue ( 21 breast cancer and no melanoma cases) containing the primary tumour and also on the bisected sentinel lymph node. 
Whole body and finger dosimeters were worn by the Pathologist during processing of the tissue for routine histopathology assessment on the day after surgery (method 1), and also measured during the taking of imprint cytology of the fresh breast specimen (method 2). A total of 21 samples were analysed.

Medical couriers: The cumulative radiation exposure for courier staff transporting the breast tissues from the operating theatre, via radiology, to the pathology laboratory was measured for 19 cases.

The cumulative radiation dose was measured using a TLD whole body waist badge worn by courier staff transporting the breast tissues in plastic containers to the pathology laboratory for 19 breast specimens.

\section{Results}

\section{Surgeons}

The cumulative whole body radiation dose to the surgeons (total 36 studies) was not detectable, being below the detection sensitivity of the dosimeter (approximately $10 \mu \mathrm{Sv}$ ).

Tables 1 and 2 provide the cumulative extremity dose to surgeons from 24 breast and melanoma studies over 6 separate measurementtime periods. These comprised 3 periods where radiation was measurable $(\mathrm{A}, \mathrm{B}, \mathrm{C})$ and 3 where radiation was not measurable $(\mathrm{D}, \mathrm{E}, \mathrm{F})$, separated for convenience into Table 1 and 2 respectively. The average extremity dose for the 3 wearing periods $(A, B, C)$ in which a radiation dose was recordable ( 8 breast and 2 melanoma) (Table 1) was $250 \mu \mathrm{Sv}$ (Table 1 and 2).

The mean time for breast procedures was 47 minutes and for melanoma 77 minutes - these were statistically significantly different ( $\mathrm{p}$ $<0.03$ ). There was no correlation between extremity dose and duration of the procedure.

\section{Radiologist}

The cumulative radiation dose to whole body and extremity for the radiology staff was not detectable using the current TLD dosimeters.

\section{Pathologists}

The cumulative whole body radiation dose for the pathologist examining the breast or melanoma samples was not detectable using the current dosimeters used during either method 1 or method 2. The extremity dose for the 9 melanoma and breast specimens processed using method 1 (on the day after surgery) was below the detection limit.

\begin{tabular}{|l|l|l|l|l|}
\hline $\begin{array}{l}\text { Wearing period } \\
(1 \text { month each })\end{array}$ & Dose $(\mu \mathrm{Sv})$ & Time $(\mathrm{min})$ & $\begin{array}{l}\text { Breast } \\
\text { cases }\end{array}$ & Melanoma cases \\
\hline A & 800 & 77 & 2 & 0 \\
\hline B & 800 & 200 & 2 & 1 \\
\hline C & 900 & 310 & 4 & 1 \\
\hline Totals & 2500 & 587 & 8 & 2 \\
\hline
\end{tabular}

Table 1: Wearing periods for finger badges in which doses for surgeons were recorded.

\begin{tabular}{|l|l|l|l|l|}
\hline $\begin{array}{l}\text { Wearing period } \\
(1 \text { month each) }\end{array}$ & Dose $(\mu \mathrm{Sv})$ & Time $(\min )$ & $\begin{array}{l}\text { Breast } \\
\text { cases }\end{array}$ & Melanoma cases \\
\hline D & 0 & 178 & 2 & 3 \\
\hline E & 0 & 500 & 1 & 6 \\
\hline F & 0 & 200 & 0 & 2 \\
\hline Totals & 0 & 878 & 3 & 11 \\
\hline
\end{tabular}

Table 2: Wearing periods for finger badges in which doses for surgeons were not measurable.
However, using method 2, the extremity dose when performing imprint cytology of the margins of the fresh breast specimen was 200 $\mu \mathrm{Sv}$ for the 21 procedures - approximately $10 \mu \mathrm{Sv}$ per procedure.

\section{Medical courier}

The cumulative whole body radiation dose for the couriers transporting the specimens was below the detection limit.

\section{Discussion}

Sentinel lymph node tracing and biopsy (LM/ SNB) techniques using radiopharmaceuticals have gradually gained status as a clinical staging and potential therapeutic tool. The widespread use of these techniques has meant that surgeons, pathologists and other staff are potentially exposed to radiation from the injected radioactive material. This has led to safety concerns for hospital staff involved in the sentinel node dissection and evaluation pathway. Although strict radiation handling and safety procedures almost universally apply in Nuclear Medicine Departments, the issues relating to the handling of radioactive materials outside of these areas may not be well understood by staff.

In our institution we have addressed requirements to assure safe work practices for staff performing LM and SNB studies. These have included tissue transportation, radiological handling and pathological processing. Although several studies have provided some important information concerning radiation exposure and possible suitable protective measures with $\mathrm{LN}$ and SNB for surgeons and pathologists [5-11], little information exists on extremity (or whole body) radiation exposure to other staff. More recent studies [12-17] have addressed parts of the LM/ SNB procedure pathway. Our studies specifically addressed the radiation exposure of staff involved in the entire LM/ SNB pathway.

\section{Dose to the operating surgeon}

A small study has demonstrated that there was no statistical difference in radiation exposure to the hands of the surgeon or first assistant between melanoma and breast cancer sentinel node biopsy procedures using ${ }^{99 \mathrm{~m}} \mathrm{Tc}$ albumin sulphur colloid [5].

Our data suggest that the extremity radiation dose to the surgeon is measurably higher for breast cancer than for melanoma LM/ SNB procedures, despite a longer time being required for melanoma cases. This can be explained by (i) the shorter distance (on average) of the sentinel node(s) from the primary tumour in breast cancer studies (thus increasing the working time of the surgeon closer to the injection site) compared to melanoma procedures, and (ii) the reduced direct contact time with the primary tumour site for excision of the melanoma primary, where forceps and longer handled instruments are commonly used, compared with that for the breast primary tumour excision, where relatively more close finger manipulation of the tumour is usual.

The extremity dose (finger, hand) to surgeons from breast studies of approximately $250 \mu \mathrm{Sv}$ per study is still low compared with annual International limits $(500,000 \mu \mathrm{Sv}$ for radiation workers and 50,000 $\mu \mathrm{Sv}$ for general public, or non-radiation workers (ICRP 60). Therefore, at conservative, minimum radiation safety levels some 200 breast cancer procedures could be performed per annum by a single surgeon before the radiation limit for non-radiation workers is exceeded - and some 2000 surgical procedures could be performed before the limit for radiation workers is exceeded.

In addition, our data show that the whole body dose to the surgeon 
from LM/ SNB is negligible - not being measurable using standard personal dosimeters over a number of studies.

\section{The dose to the radiologist}

Specimen mammography and/ or ultrasound of the surgical breast specimen is now commonplace for assessment of radiological margins to predict the adequacy of surgical clearance. This has meant that imaging staff may be exposed to radiation from ${ }^{99 \mathrm{~m}} \mathrm{Tc}$ tracers used for LM/ SNB during specimen radiography. However, our data show that whole body or extremity radiation exposure to radiology staff is negligible.

\section{The dose to the pathologist}

When the pathologist processed the primary melanoma specimen the day after LM/ SNB procedure, sufficient decay had occurred to reduce the radioactivity substantially, so that no whole body or extremity dose was detected. However, if the tissue was processed on the day of surgery, for example if performing breast tissue imprint cytology, the pathologist received an extremity dose of approximately $10 \mu \mathrm{Sv}$ per procedure. Imprint cytology for margin assessment is not routinely practiced in most institutions. The examination of SN imprint cytology and the handling of the breast specimen after fixation were associated with still lower exposure levels.

\section{The dose to specimen transport and laboratory staff}

Staff transporting the radioactive specimen from the operating theatre to the radiology suite and/ or pathology laboratory are potentially at risk of radiation exposure from holding the specimen container close to their bodies. This could be further increased if several radioactive specimens were transported together. Measurement of whole body doses revealed negligible radiation exposure when couriers carried radioactive materials by the handle of a standard bucket-type plastic specimen container.

\section{The dose to nursing and ancillary staff}

As radiation exposureis inversely proportional to the square of the distance from the source (inverse square law), exposure during LM/ SNB procedures for nursing staff and other staff, including anaesthetic and observing surgical staff, is considerably less than for the surgeon. Distances beyond 1-2 meters ensure that the radiation dose received by staff away from the operating table in these procedures is negligible.

The extremity dose to nursing staff was not measured in this study. However, as nursing staff routinely use long forceps to transfer radioactive specimens from the operating table into specimen containers for transport, and as the transfer time is very short, the dose would be negligible.

\section{Dose to the nuclear medicine personnel}

The major source of radiation exposure to nuclear medicine staff is from close proximity to the concentrated radionuclide prior to injection into the patient. Further potential risk occurs from leakage of radionuclide from the skin injection sites onto skin, clothing or bedlinen. Safe handling guidelines for radioactive materials are in routine use in nuclear medicine departments.

Our results demonstrate that LM/ SNB techniques are safe for surgeons, pathologists, radiologists and courier staff who are potentially at risk of radiation exposure from these sources and confirm the findings of the few studies in the literature.

\section{Conclusions}

Whole body dose to the surgeon, couriers, radiology staff and pathologist LM/ SNB is extremely low.

Some 200 (at maximum some 2000) breast cancer procedures to be performed per annum by a single surgeon before standard allowable limits are exceeded.

The extremity radiation exposure for melanoma LM/ SNB procedures appears much lower than for breast cancer LM/ SNB procedures.

Extremity doses for radiology, pathology, courier and other staff are much lower than for surgeons and are essentially negligible using safe work practices.

In conclusion, even though the radiation levels are low for LM/ $\mathrm{SNB}$, good radiation handling practices should still be adopted in all cases during surgery, specimen transportation and pathological processing to ensure that the ALARA (As Low as Reasonably Achievable) Principle of radiation exposure is applied. In general terms, it is good practice for staff who are pregnant (or potentially so) to avoid any procedure involving irradiation, and exercise extra care to reduce avoidable radiation exposure risk (Table 3).

Some Basic Radiation Safety Recommendations for Lymphatic Mapping and Sentinel Node Biopsy

Although the radiation levels measured in this study were very low, detailed safety protocols should be in place and practiced to ensure that staff are exposed to the minimum levels of radiation in LM/ SNB procedures and tissue processing. If such procedures are followed lead aprons, which are often heavy, cumbersome and may produce skeletal strain, are not required.

Some basic information that could be incorporated into LM/ SNB protocols is given below:

\section{Surgeon and ancillary staff}

The dose to the surgeon's hands can be effectively reduced by a factor of about 30 with the use of $20 \mathrm{~cm}$ forceps, artery clips, pencil electrocautery and other instruments, since radiation exposure varies inversely with the square of distance from the source.

Ensuring only a brief duration of contact or limited time spent within a $10 \mathrm{~cm}(4 \mathrm{inch})$ radius of the injection site can further reduce radiation exposure. Awareness of these points by staff can ensure the best practice during LM/ SNB procedures. If possible the primary tumour (which contains $>95 \%$ of the radioactive dose) should be removed as soon as possible after the sentinel node(s) have been

\begin{tabular}{|l|l|l|}
\hline STAFF MEMBER & DOSE PER CASE & MAX CASE NO. ${ }^{*}$ \\
\hline Surgeon - melanoma case & $0 \mu \mathrm{Sv}$ & Effectively unlimited \\
\hline Surgeon- breast case & $250 \mu \mathrm{Sv}$ & $200^{\dagger}$ \\
\hline Radiologist & $0 \mu \mathrm{Sv}$ & Effectively unlimited $^{+}$ \\
\hline Pathologist (same day) & $10 \mu \mathrm{Sv}$ & $5000^{\dagger}$ \\
\hline Pathologist (next day) & $0 \mu \mathrm{Sv}$ & Effectively unlimited \\
\hline Medical Courier & $0 \mu \mathrm{Sv}$ & Effectively unlimited \\
\hline
\end{tabular}

*Based on a radiation safety limit of $50,000 \mu \mathrm{Sv}$ for general public (for non-radiation workers). ${ }^{\dagger}$ Calculated using the minimum radiation dose limit for non-radiation workers; 2000 cases if the minimum radiation dose limit for radiation workers is used

Table 3: Estimated radiation safety limits for LM and SNB procedures for staff. 
excised, and radioactive materials should be placed $>2$ metres away from staff.

\section{Radiographer and radiologist}

The dose to the radiologist and radiographer can be reduced by minimal direct handling and rapid processing of the specimen.

\section{Pathologist and pathology technician}

The dose to the pathologist can be substantially reduced if the pathology processing of the primary tumour is delayed for 24 hours, since radioactivity from ${ }^{99 \mathrm{~m}} \mathrm{Tc}$ is halved every 6 hours (1/16 at $24 \mathrm{hr}$ ). Urgent specimens can be safely handled immediately using longhandled instruments and similar precautions to that used by surgeons. It is not necessary to delay processing of the sentinel node.

\section{Specimen transport and laboratory personnel}

The excised specimen should be placed in a container with a handle and with a prominently displayed radioactive substance label. The courier should carry the container using the handle, away from their body, and it should be transported expediently to the laboratory. Suitable protocols for handling of radioactive substances should be in place and actively practiced by laboratory staff to safely store and process pathological specimens.

\section{Nuclear medicine and ward staff}

Spillage and leakage of radioactive material from the injection site can be minimised by careful injection technique with the site draped and standard isotope handling protocols. Taping a swab over the injection site after completion of the isotope injection can contain any inadvertent leakage, preventing soiling of bed-linen. This technique also assists the surgeon in confirming that the agreed primary tumour site has been accurately injected.

\section{Waste disposal}

Linen, gauze swabs and other potentially low-level ${ }^{99 \mathrm{~m}} \mathrm{Tc}$ contaminated material can usually be disposed of in the same way as other non-radioactive biological waste. Human tissue samples containing higher radioactivity can be processed, stored or disposed of in standard methods to that used for non-radioactive biological waste after 24-48 hours has elapsed from the initial radiopharmaceutical injection time - this time period permits adequate radioactive decay of ${ }^{99 \mathrm{~m}} \mathrm{Tc}$.

\section{Acknowledgements}

The authors thank Dr Tom Dodd, Consultant Pathologist, IMVS, Dr T Bessen, Radiology for assisting in coordination of the pathology and radiology studies respectively.

\section{References}

1. Keshtgar MRS, Waddington WA, Lakhani SR, Ell PJ (1999) The sentinel node in surgical oncology. Chapter 8 Dosimetry and radiation protection. Springer, London, Pages 92-101.

2. Morton DL, Wen DR, Wong JH, Economou JS, Cagle LA, et al. (1992) Technica details of intraoperative lymphatic mapping for early stage melanoma. Arch Surg 127: 392-399.
3. Morton DL, Thompson JF, Essner R, Elashoff R, Stern SL, et al. (1999) Validation of the accuracy of intraoperative lymphatic mapping and sentine lymphadenectomy for early-stage melanoma: a multicenter trial. Multicenter Selective Lymphadenectomy Trial Group. Ann Surg 230: 453-463; discussion 463-465.

4. Krag DN, Weaver DL, Alex JC, Fairbank JT (1993) Surgical resection and radio-localization of the sentinel lymph node in breast cancer using a gamma probe. Surg Oncol 2: 335-339; discussion 340.

5. Miner TJ, Shriver CD, Jaques DP, Maniscalco-Theberge ME, Krag DN (1999) Sentinel lymph node biopsy for breast cancer: the role of previous biopsy on patient eligibility. Am Surg 65: 493-498; discussion 498-499.

6. Stratmann SL, McCarty TM, Kuhn JA (1999) Radiation safety with breast sentinel node biopsy. Am J Surg 178: 454-457.

7. Cremonesi M, Ferrari M, Sacco E, Rossi A, De Cicco C, et al. (1999) Radiation protection in radioguided surgery of breast cancer. Nucl Med Commun 20: 919 924.

8. Cibull ML (1999) Handling sentinel lymph node biopsy specimens. A work in progress. Arch Pathol Lab Med 123: 620-621.

9. Glass EC, Basinski JE, Krasne DL, Giuliano AE (1999) Radiation safety considerations for sentinel node techniques. Ann Surg Oncol 6: 10-11.

10. Fitzgibbons PL, LiVolsi VA (2000) Recommendations for handling radioactive specimens obtained by sentinel lymphadenectomy. Surgical Pathology Committee of the College of American Pathologists, and the Association of Directors of Anatomic and Surgical Pathology. Am J Surg Pathol 24: 15491551.

11. Waddington WA, Keshtgar MR, Taylor I, Lakhani SR, Short MD, et al. (2000) Radiation safety of the sentinel lymph node technique in breast cancer. Eur J Nucl Med 27: 377-391

12. Morton R, Horton PW, Peet DJ, Kissin MW (2003) Quantitative assessmen of the radiation hazards and risks in sentinel node procedures. $\mathrm{Br} \mathrm{J}$ Radiol 76 : $117-122$

13. de Kanter AY, Arends PP, Eggermont AM, Wiggers T (2003) Radiation protection for the sentinel node procedure in breast cancer. Eur J Surg Oncol 29:396-399.

14. Koizumi M, Nomura E, Yamada Y, Takiguchi T, Tanaka K et al (2003) Sentine node detection using $99 \mathrm{mTc}$-rhenium sulphide colloid in breast cancer patients: evaluation of 1 day and 2day protocols, and a dose-finding study. Nucl Med Commun 24: 663-670.

15. Klausen TL, Chakera AH, Friis E, Rank F, Hesse B, et al (2005) Radiation doses to staff involved in sentinel node operations for breast cancer. Clin Physiol Funct Imagin 25: 196-202.

16. Renshaw AA, Kish R, Gould EW (2010) Increasing radiation from sentinel node specimens in pathology over time. Am J Clin Pathol 134: 299-302.

17. Singleton M, Firth M, Stephenson T, Morrison G, Baginska J (2012) Radiationguided breast sentinel lymph node biopsies - is a handling delay for radiation protection necessary? Histopathology.
This article was originally published in a special issue, Surgical oncology: Clinical Importance handled by Editor(s). Dr. Liqiang Zhang, Arizona State University, USA; Dr. Salomone Di Saverio, Surgery and Trauma Surgery Unit Italy 